\title{
Pacific
}

Journal of

Mathematics

\section{REGULARITY OF CANONICAL AND DEFICIENCY MODULES FOR MONOMIAL IDEALS}

\author{
Manoj Kummini and Satoshi Murai
}




\title{
REGULARITY OF CANONICAL AND DEFICIENCY MODULES FOR MONOMIAL IDEALS
}

\author{
Manoj Kummini And SATOShi MURAi
}

\begin{abstract}
We show that the Castelnuovo-Mumford regularity of the canonical or a deficiency module of the quotient of a polynomial ring by a monomial ideal is bounded by its dimension.
\end{abstract}

\section{Introduction}

Let $R=\mathbb{k}\left[x_{1}, \ldots, x_{n}\right]$ be a standard graded polynomial ring over a field $\mathbb{k}$, and let $\mathfrak{m}=\left(x_{1}, \ldots, x_{n}\right)$ be the homogeneous maximal ideal of $R$. We study the Castelnuovo-Mumford regularity of the modules $\operatorname{Ext}_{R}^{i}\left(R / I, \omega_{R}\right)$ when $I \subset R$ is a monomial ideal; here $\omega_{R}=R(-n)$ denotes the canonical module of $R$. The modules

$$
\operatorname{Ext}_{R}^{i}\left(R / I, \omega_{R}\right) \text { for } i>n-\operatorname{dim} R / I
$$

are called the deficiency modules of $R / I$, while

$$
\operatorname{Ext}_{R}^{n-\operatorname{dim} R / I}\left(R / I, \omega_{R}\right)
$$

is called the canonical module of $R / I$.

For any homogeneous ideal $I \subseteq R$, the local cohomology modules $\mathrm{H}_{\mathfrak{m}}^{i}(R / I)$ are important in commutative algebra and algebraic geometry. One is often interested in the vanishing of homogeneous components of $\mathrm{H}_{\mathfrak{m}}^{i}(R / I)$. While one cannot expect the vanishing of $\mathrm{H}_{\mathfrak{m}}^{i}(R / I)$ in negative degrees (unless it has finite length), one can, using the local duality theorem of Grothendieck, obtain some information from $\operatorname{Ext}_{R}^{n-i}\left(R / I, \omega_{R}\right)$. For a finitely generated graded $R$-module $M$, its (CastelnuovoMumford) regularity $\operatorname{reg}(M)$ is an invariant that contains information about the stability of homogeneous components in sufficiently large degrees. In light of these, it is desirable to get bounds on $\operatorname{reg}\left(\operatorname{Ext}_{R}^{i}\left(R / I, \omega_{R}\right)\right)$. Such bounds were studied by L. T. Hoa and E. Hyry [2006] and by M. Chardin, D. T. Ha and Hoa [2009]; see also the references in those papers.

Unfortunately, canonical and deficiency modules can have large regularity. For a finitely generated graded $R$-module $M$, known bounds for $\operatorname{reg}\left(\operatorname{Ext}_{R}^{i}\left(M, \omega_{R}\right)\right)$

MSC2000: 13D07, 13D45.

Keywords: canonical modules, Castelnuovo-Mumford regularity. 
are large; see, for example, [Hoa and Hyry 2006, Theorems 9 and 14]. On the other hand, more optimal bounds for $\operatorname{reg}\left(\operatorname{Ext}_{R}^{i}\left(R / I, \omega_{R}\right)\right)$ are known to exist for certain classes of graded ideals $I$; see [Hoa and Hyry 2006, Section 4]. It is an interesting problem to find a class of graded ideals $I \subset R$ with optimal bounds for $\operatorname{reg}\left(\operatorname{Ext}_{R}^{i}\left(R / I, \omega_{R}\right)\right)$. In this paper, we focus on monomial ideals. It follows from the theory of square-free modules, introduced by K. Yanagawa [2000], that if $I$ is a square-free monomial ideal, then $\operatorname{reg}\left(\operatorname{Ext}_{R}^{i}\left(R / I, \omega_{R}\right)\right) \leq \operatorname{dim} \operatorname{Ext}_{R}^{i}\left(R / I, \omega_{R}\right)$. This bound is small, since $\operatorname{dim} \operatorname{Ext}_{R}^{i}\left(R / I, \omega_{R}\right) \leq n-i$; see [Bruns and Herzog 1993, Corollary 3.5.11].

While one cannot apply the theory of square-free modules to all monomial ideals, there are results that show that $\operatorname{reg}\left(\operatorname{Ext}_{R}^{i}\left(R / I, \omega_{R}\right)\right)$ is not large when $I$ is a monomial ideal. For example, we see from [Takayama 2005, Proposition 1, page 333] that if $\operatorname{Ext}_{R}^{i}\left(R / I, \omega_{R}\right)$ has finite length, then its regularity is negative or equal to zero. Again, Hoa and Hyry [2006, Proposition 21] showed that if $\mathrm{H}_{\mathfrak{m}}^{i}(R / I)$ has finite length for $i=0,1, \ldots, d-1$, where $d=\operatorname{dim} R / I$, then $\operatorname{reg}\left(\operatorname{Ext}_{R}^{n-d}\left(R / I, \omega_{R}\right)\right) \leq d$. We generalize these results in the next theorem:

Theorem 1.1. Let $I \subseteq R$ be a monomial ideal. Then

$$
\operatorname{reg}\left(\operatorname{Ext}_{R}^{i}\left(R / I, \omega_{R}\right)\right) \leq \operatorname{dim} \operatorname{Ext}_{R}^{i}\left(R / I, \omega_{R}\right) \quad \text { for all } 0 \leq i \leq n
$$

Since $\operatorname{dim} \operatorname{Ext}_{R}^{i}\left(R / I, \omega_{R}\right) \leq n-i$, we immediately get this:

Corollary 1.2. Let $I \subseteq R$ be a monomial ideal. Then

$$
\operatorname{reg}\left(\operatorname{Ext}_{R}^{i}\left(R / I, \omega_{R}\right)\right) \leq n-i \quad \text { for all } 0 \leq i \leq n \text {. }
$$

In general, this conclusion need not hold without the assumption that $I$ is a monomial ideal; see [Chardin and D'Cruz 2003, Example 3.5].

Our approach to bounding the regularity of canonical and deficiency modules differs from that of Hoa and Hyry. We show that if $I$ is a monomial ideal, then $\operatorname{Ext}_{R}^{i}\left(R / I, \omega_{R}\right)$ has a multigraded filtration, called the Stanley filtration and introduced by D. Maclagan and G. G. Smith [2005]; the bound on regularity follows from this filtration.

In the next section, we discuss some preliminaries on Stanley filtrations and local cohomology. In Section 3, we prove our main result.

\section{Preliminaries}

Hereafter we take $R$-modules to be graded by $\mathbb{Z}^{n}$, giving deg $x_{i}=\boldsymbol{e}_{i}$, the $i$-th unit vector of $\mathbb{Z}^{n}$. We call this the multigrading of $R$ and $R$-modules. 
Notation 2.1. Let $\boldsymbol{a}=\left(a_{1}, \ldots, a_{n}\right) \in \mathbb{Z}^{n}$. Write

$$
\boldsymbol{x}^{\boldsymbol{a}}=\prod_{i=1}^{n} x_{i}^{a_{i}} \in \mathbb{k}\left[x_{1}^{ \pm 1}, \ldots, x_{n}^{ \pm 1}\right] .
$$

We say that $\boldsymbol{a}$ is the degree of $\boldsymbol{x}^{a}$ and write $\operatorname{deg} \boldsymbol{x}^{\boldsymbol{a}}=\boldsymbol{a}$. Define $\operatorname{Supp}(\boldsymbol{a})=\left\{i: a_{i} \neq 0\right\}$, and define $\boldsymbol{a}^{+}, \boldsymbol{a}^{-} \in \mathbb{N}^{n}$ by the conditions

$$
\boldsymbol{a}=\boldsymbol{a}^{+}-\boldsymbol{a}^{-} \quad \text { and } \quad \operatorname{Supp}\left(\boldsymbol{a}^{+}\right) \cap \operatorname{Supp}\left(\boldsymbol{a}^{-}\right)=\varnothing .
$$

Write $\|\boldsymbol{a}\|$ for $\sum_{i=1}^{n} a_{i}$, the total degree of $\boldsymbol{a}$ (and of the monomial $\boldsymbol{x}^{\boldsymbol{a}}$ ). We will say that $\boldsymbol{a}$ (or equivalently $\boldsymbol{x}^{a}$ ) is square-free if $a_{i} \in\{0,1\}$ for all $i$. Let $[n]=\{1, \ldots, n\}$. For $\Lambda \subseteq[n]$, we set $\boldsymbol{e}_{\Lambda}=\sum_{i \in \Lambda} \boldsymbol{e}_{i}$ and abbreviate the (square-free) monomial $\boldsymbol{x}_{\Lambda}^{\boldsymbol{e}_{\Lambda}}$ as $x_{\Lambda}$. The canonical module of $R$ is $\omega_{R}=R\left(-\boldsymbol{e}_{[n]}\right)$.

Let $M$ be a finitely generated multigraded $R$-module. Let $m \in M$ be a homogeneous element, and let $G \subset\left\{x_{1}, \ldots, x_{n}\right\}$ be a subset such that $u m \neq 0$ for all monomials $u \in \mathbb{k}[G]$. The $\mathbb{k}$-subspace $\mathbb{k}[G] m$ of $M$ generated by all the $u m$, where $u$ is a monomial in $\mathbb{k}[G]$, is called a Stanley space. A Stanley decomposition of $M$ is a finite set $\mathscr{Y}$ of pairs $(m, G)$ of homogeneous elements $m \in M$ and $G \subseteq\left\{x_{1}, \ldots, x_{n}\right\}$ such that $\mathbb{k}[G] m$ is a Stanley space for all $(m, G) \in \mathscr{Y}$ and

$$
M={ }_{\mathbb{k}} \bigoplus_{(m, G) \in \mathscr{S}} \mathbb{k}[G] m .
$$

(We used "= ${ }_{\mathbb{k}}$ " to emphasize that the decomposition is only as vector spaces.) Properties of such decompositions have been widely studied; we follow the approach of [Maclagan and Smith 2005, Section 3], where Stanley decompositions were used to get bounds for multigraded regularity. Following [Maclagan and Smith 2005, Definition 3.7], we define a Stanley filtration to be a Stanley decomposition with an ordering of pairs $\left\{\left(m_{i}, G_{i}\right): 1 \leq i \leq p\right\}$ such that

$$
\left(\sum_{i=1}^{j} R m_{i}\right) /\left(\sum_{i=1}^{j-1} R m_{i}\right)=\mathbb{k}\left[G_{j}\right]\left(-\operatorname{deg} m_{j}\right) \text { for } j=1,2, \ldots, p
$$

as $R$-modules. Note, in this case, that

$$
0 \subseteq R m_{1} \subseteq \cdots \subseteq \sum_{i=1}^{j} R m_{i} \subseteq \cdots \subseteq \sum_{i=1}^{p} R m_{i}=M
$$

is a prime filtration of $M$, as in [Eisenbud 1995, Proposition 3.7, page 93].

Proposition 2.2. Let $M$ be a multigraded $R$-module with a Stanley decomposition $\mathscr{Y}$ such that $(\operatorname{deg} m)^{+}$is square-free and $G=\operatorname{Supp}\left((\operatorname{deg} m)^{+}\right)$for all $(m, G) \in \mathscr{Y}$. Then, $\mathscr{Y}$ gives a Stanley filtration. Moreover, $\operatorname{reg} M \leq \max \{\|\operatorname{deg} m\|:(m, G) \in \mathscr{Y}\}$. 
Proof. We order $\mathscr{Y}=\left\{\left(m_{1}, G_{1}\right), \ldots,\left(m_{p}, G_{p}\right)\right\}$ so that $\left\|\operatorname{deg} m_{1}\right\| \geq \cdots \geq\left\|\operatorname{deg} m_{p}\right\|$. It follows from our hypothesis that

$$
\operatorname{span}_{\mathbb{k}}\left\{m_{1}, \ldots, m_{p}\right\}=\operatorname{span}_{\mathbb{k}}\left\{m \in M: \operatorname{Supp}\left((\operatorname{deg} m)^{+}\right) \text {is square-free }\right\},
$$

where $\operatorname{span}_{\mathbb{k}}(V)$ denotes the $\mathbb{k}$-vector space spanned by the elements in $V$. We write $M^{(j)}$ for $\sum_{i=1}^{j} R m_{i}$. We will now show, inductively on $j$, that

(a) $M^{(j-1)}:_{R} m_{j}=\left(x_{k} ; x_{k} \notin G_{j}\right)$, and

(b) the set $\bigcup_{i=1}^{j}\left\{u m_{i}: u\right.$ is a monomial in $\left.\mathbb{k}\left[G_{i}\right]\right\}$ is a $\mathbb{k}$-basis for $M^{(j)}$.

These imply that $\mathscr{Y}$ is a Stanley filtration of $M$.

Let $j=1$. We will show that $\left(0:_{R} m_{1}\right)=\left(x_{k} ; x_{k} \notin G_{1}\right)$. We have $u m_{1} \neq 0$ for all monomials $u \in \mathbb{k}\left[G_{1}\right]$ from the definition of the decomposition. Therefore we must show that $x_{l} m_{1}=0$ for any $x_{l} \notin G_{1}$. Let $x_{l} \notin G_{1}$. Then $\left(\operatorname{deg} x_{l} m_{1}\right)^{+}$ is square-free, and $x_{l} m_{1} \in \operatorname{span}_{\mathbb{k}}\left\{m_{1}, \ldots, m_{p}\right\}$ by (2). However, from the choice of $m_{1}$, we see that $x_{l} m_{1}=0$. Therefore $\left(0:_{R} m_{1}\right)=\left(x_{k} ; k \notin G_{1}\right)$, proving (a). Then (b) follows immediately.

Now, assume that $j>1$ and that the assertion is known for all $i<j$. We first show (a). Let $u$ be a monomial in $\mathbb{k}\left[G_{j}\right]$. By statement (b) for $j-1$, the set $\bigcup_{i=1}^{j-1}\left\{v m_{i}: v\right.$ is a monomial in $\left.\mathbb{k}\left[G_{i}\right]\right\}$ is a $\mathbb{k}$-basis for $M^{(j-1)}$. Since $u m_{j}$ is an element of the basis of $M$ coming from the Stanley decomposition, $u m_{j}$ is not in the $\mathbb{k}$-linear span of $\bigcup_{i=1}^{j-1}\left\{v m_{i}: v\right.$ is a monomial in $\left.\mathbb{k}\left[G_{i}\right]\right\}$, that is, $u m_{j} \notin M^{(j-1)}$. It remains to prove that $x_{l} m_{j} \in M^{(j-1)}$ for any $x_{l} \notin G_{j}$. Let $x_{l} \notin G_{j}$. Since $\left(\operatorname{deg} x_{l} m_{j}\right)^{+}$is square-free, it follows from (2) and the ordering of the $\left(m_{i}, G_{i}\right)$ that

$$
x_{l} m_{j} \in \operatorname{span}_{\mathbb{k}}\left\{m_{i}: 1 \leq i \leq p, \operatorname{deg} m_{i}>\operatorname{deg} m_{j}\right\} \subseteq \operatorname{span}_{\mathbb{k}}\left\{m_{1}, \ldots, m_{j-1}\right\} .
$$

Therefore $x_{l} m_{j} \in M^{(j-1)}$, proving the statement (a) for $j$.

From (a), we see that the sequence

$$
0 \rightarrow M^{(j-1)} \rightarrow M^{(j)} \rightarrow \mathbb{k}\left[G_{j}\right] m_{j} \rightarrow 0
$$

is exact. Now, statement (b) for $j$ follows from the induction hypothesis.

Theorem 4.1 of [Maclagan and Smith 2005] essentially gives the assertion about regularity, but we give a quick proof here by showing that

$$
\operatorname{reg} M^{(j)} \leq \max \left\{\left\|\operatorname{deg} m_{i}\right\|: 1 \leq i \leq j\right\} \quad \text { for all } 1 \leq j \leq p .
$$

It holds for $j=1$. For $j>1$, it follows from [Eisenbud 1995, Corollary 20.19] and the exact sequence (3) that

$$
\operatorname{reg} M^{(j)} \leq \max \left\{\operatorname{reg} M^{(j-1)},\left\|\operatorname{deg} m_{j}\right\|\right\} .
$$

Then induction completes the proof. 
Finally, we recall some basics of local cohomology. We follow [Bruns and Herzog 1993, Sections 3.5 and 3.6]. Let $\check{C}^{\bullet}$ be the Čech complex on $x_{1}, \ldots, x_{n}$; the term at the $i$-th cohomological degree is

$$
\check{C}^{i}=\bigoplus_{\Lambda \subseteq[n],|\Lambda|=i} R_{x_{\Lambda}}
$$

where $R_{x_{\Lambda}}$ denotes inverting the monomial $x_{\Lambda}$. Note that $\check{C}^{\bullet}$ is a complex of $\mathbb{Z}^{n}$-graded $R$-modules, with differentials of degree 0 . For a finitely generated $R$ module $M$, we set $\check{C}^{\bullet}(M)=\check{C}^{\bullet} \otimes_{R}(M)$. Then $\mathrm{H}_{\mathfrak{m}}^{i}(M)=\mathrm{H}^{i}\left(\check{C}^{\bullet}(M)\right)$.

Definition 2.3. Let $F \subseteq[n]$. We define $\check{C}_{F}^{\bullet}$ to be the subcomplex of $\check{C}^{\bullet}$ obtained by setting

$$
\check{C}_{F}^{i}=\left\{\begin{array}{cl}
0 & \text { if } i<|F|, \\
\bigoplus_{F \subseteq \Lambda \subseteq[n]} R_{x_{\Lambda}} & \text { otherwise. }
\end{array}\right.
$$

Lemma 2.4. Let I be a monomial ideal and $F \subseteq[n]$. If $\boldsymbol{a} \in \mathbb{Z}^{n}$ is such that $\operatorname{Supp}\left(\boldsymbol{a}^{-}\right)=F$, then $\mathrm{H}_{\mathfrak{m}}^{i}(R / I)_{\boldsymbol{a}}=\mathrm{H}^{i}\left(\check{C}_{F}^{\bullet} \otimes_{R}(R / I)\right)_{\boldsymbol{a}}$.

Proof. The proof of [Takayama 2005, Theorem 1] uses this argument implicitly. Since $\mathrm{H}_{\mathfrak{m}}^{i}(R / I)_{\boldsymbol{a}}=\mathrm{H}^{i}\left(\left(\check{C}^{\bullet}(R / I)\right)_{\boldsymbol{a}}\right.$, it suffices to show that

$$
\left(\check{C}^{\bullet}(R / I)\right)_{\boldsymbol{a}}=\left(\check{C}_{F}^{\bullet} \otimes_{R}(R / I)\right)_{\boldsymbol{a}} .
$$

This, in turn, stems from the fact that $\check{C}_{F}^{j} \otimes_{R}(R / I)$ consists precisely of the direct summands of $\check{C}^{j}(R / I)$ that are nonzero in multidegree $\boldsymbol{a}$ for all $1 \leq j \leq n$.

\section{Proof of the main theorem}

Lemma 3.1. Let $I \subset R$ be a monomial ideal. Let $\boldsymbol{a} \in \mathbb{Z}^{n}$ and $j \in \operatorname{Supp}\left(\boldsymbol{a}^{+}\right)$. The multiplication map

is bijective.

$$
x_{j}: \operatorname{Ext}_{R}^{i}\left(R / I, \omega_{R}\right)_{a} \rightarrow \operatorname{Ext}_{R}^{i}\left(R / I, \omega_{R}\right)_{a+e_{j}}
$$

Proof. We first claim that the multiplication map

$$
x_{j}: \mathrm{H}_{\mathfrak{m}}^{n-i}(R / I)_{-a-e_{j}} \rightarrow \mathrm{H}_{\mathfrak{m}}^{n-i}(R / I)_{-a}
$$

is bijective. By local duality [Bruns and Herzog 1993, Theorem 3.6.19], this map is the Matlis dual of the multiplication by $x_{j}$ on $\operatorname{Ext}_{R}^{i}\left(R / I, \omega_{R}\right)_{a}$; hence, it suffices to prove the claim above.

Set $F=\operatorname{Supp}\left(\boldsymbol{a}^{+}\right)$. Note that $\operatorname{Supp}\left(\boldsymbol{a}^{+}+\boldsymbol{e}_{j}\right)=F$. For all $i$, the map $x_{j}$ acts as a unit on $\check{C}_{F}^{i}$. Therefore the homomorphism of complexes

$$
\check{C}_{F}^{\bullet} \otimes_{R}(R / I) \rightarrow \check{C}_{F}^{\bullet} \otimes_{R}(R / I)
$$


induced by the multiplication map $x_{j}: \check{C}_{F}^{i} \otimes_{R}(R / I) \rightarrow \check{C}_{F}^{i} \otimes_{R}(R / I)$ is an isomorphism. The claim now follows from Lemma 2.4 , which implies that

$$
\begin{aligned}
\mathrm{H}_{\mathfrak{m}}^{i}(R / I)_{-a-e_{j}} & =\mathrm{H}^{i}\left(\check{C}_{F}^{\bullet} \otimes_{R}(R / I)\right)_{-a-e_{j}}, \\
\mathrm{H}_{\mathfrak{m}}^{i}(R / I)_{-\boldsymbol{a}} & =\mathrm{H}^{i}\left(\check{C}_{F}^{\bullet} \otimes_{R}(R / I)\right)_{-\boldsymbol{a}} .
\end{aligned}
$$

The previous lemma says that, if $I$ is a monomial ideal, then $\operatorname{Ext}_{R}^{i}\left(R / I, \omega_{R}\right)$ is a $(1,1, \ldots, 1)$-determined module in the sense of [Miller 2000, Definition 2.1]. Proof of Theorem 1.1. For $F \subseteq[n]$, let $M_{F}^{i}$ be a multigraded $\mathbb{k}$-basis for

$$
\bigoplus_{\boldsymbol{a} \in \mathbb{N}^{n}, \operatorname{Supp}(\boldsymbol{a}) \cap F=\varnothing} \operatorname{Ext}_{R}^{i}\left(R / I, \omega_{R}\right)_{\boldsymbol{e}_{F}-\boldsymbol{a}}
$$

Let $\mathscr{Y}_{i}=\left\{(m, F): F \subseteq[n]\right.$ and $\left.m \in \mathcal{M}_{F}^{i}\right\}$. It follows from Lemma 3.1 that $\mathscr{Y}_{i}$ is a Stanley decomposition of $\operatorname{Ext}_{R}^{i}\left(R / I, \omega_{R}\right)$. In particular,

$$
\operatorname{dim} \operatorname{Ext}^{i}\left(R / I, \omega_{R}\right)=\max \left\{|F|: M_{F}^{i} \neq \varnothing\right\} .
$$

By the construction of $\mathcal{M}_{F}^{i}$, this Stanley decomposition satisfies the assumption of Proposition 2.2. Therefore

$$
\begin{aligned}
\operatorname{reg}\left(\operatorname{Ext}_{R}^{i}\left(R / I, \omega_{R}\right)\right) & \leq \max _{F \subseteq[n]}\left\{\max \left\{\|\operatorname{deg} m\|: m \in M_{F}^{i}\right\}\right\} \\
& \leq \max _{F \subseteq[n]}\left\{|F|: M_{F}^{i} \neq \varnothing\right\} \\
& =\operatorname{dim} \operatorname{Ext}_{R}^{i}\left(R / I, \omega_{R}\right),
\end{aligned}
$$

as desired. (The second inequality follows since $\|\operatorname{deg} u\|=|F|-\left\|(\operatorname{deg} u)^{-}\right\|$for any $u \in M_{F}^{i}$.)

We remark that, by using [Takayama 2005, Theorem 1] and local duality, one can determine whether $\mu_{F}^{i} \neq \varnothing$ from certain subcomplexes of the Stanley-Reisner complex of the radical $\sqrt{I}$ of $I$.

\section{Acknowledgments.}

The authors thank B. Ulrich for helpful comments. This paper was written when Murai was visiting Purdue University in September 2009. He would like to thank his host, G. Caviglia, for his hospitality.

\section{References}

[Bruns and Herzog 1993] W. Bruns and J. Herzog, Cohen-Macaulay rings, Cambridge Studies in Advanced Mathematics 39, Cambridge University Press, 1993. MR 95h:13020 Zbl 0788.13005 
[Chardin and D'Cruz 2003] M. Chardin and C. D'Cruz, "Castelnuovo-Mumford regularity: Examples of curves and surfaces", J. Algebra 270:1 (2003), 347-360. MR 2004m:13036 Zbl 1056.14065

[Chardin et al. 2009] M. Chardin, D. T. Ha, and L. T. Hoa, "Castelnuovo-Mumford regularity of Ext modules and homological degree", preprint, 2009. arXiv 0903.4535

[Eisenbud 1995] D. Eisenbud, Commutative algebra, Graduate Texts in Mathematics 150, Springer, New York, 1995. MR 97a:13001 Zbl 0819.13001

[Hoa and Hyry 2006] L. T. Hoa and E. Hyry, "Castelnuovo-Mumford regularity of canonical and deficiency modules", J. Algebra 305:2 (2006), 877-900. MR 2007g:13023 Zbl 1108.13017

[Maclagan and Smith 2005] D. Maclagan and G. G. Smith, "Uniform bounds on multigraded regularity”, J. Algebraic Geom. 14:1 (2005), 137-164. MR 2005g:14098 Zbl 1070.14006

[Miller 2000] E. Miller, "The Alexander duality functors and local duality with monomial support", J. Algebra 231:1 (2000), 180-234. MR 2001k:13028 Zbl 0968.13009

[Takayama 2005] Y. Takayama, "Combinatorial characterizations of generalized Cohen-Macaulay monomial ideals”, Bull. Math. Soc. Sci. Math. Roumanie (N.S.) 48:3 (2005), 327-344. MR 2006e: 13017 Zbl 1092.13020

[Yanagawa 2000] K. Yanagawa, "Alexander duality for Stanley-Reisner rings and squarefree $\mathbb{N}^{n}$ graded modules", J. Algebra 225:2 (2000), 630-645. MR 2000m:13036 Zbl 0981.13011

Received October 11, 2009. Revised June 7, 2010.

MANOJ KUMMINI

PURDUE UNIVERSITY

DEPARTMENT OF MATHEMATICS

150 N. UNIVERSITY ST.

WEST LAFAYETTE, IN 47907

UNITED STATES

nkummini@math.purdue.edu

SATOSHI MURAI

YAMAGUCHI UNIVERSITY

DEPARTMENT OF MATHEMATICAL SCIENCE

YAMAGUCHI 753-8512

JAPAN

murai@yamaguchi-u.ac.jp 


\title{
PACIFIC JOURNAL OF MATHEMATICS
}

\author{
http://www.pjmath.org \\ Founded in 1951 by
}

E. F. Beckenbach (1906-1982) and F. Wolf (1904-1989)

\section{EDITORS}

V. S. Varadarajan (Managing Editor)

Department of Mathematics

University of California

Los Angeles, CA 90095-1555

pacific@math.ucla.edu

Vyjayanthi Chari

Department of Mathematics

University of California

Riverside, CA 92521-0135

chari@math.ucr.edu

\section{Robert Finn}

Department of Mathematics Stanford University

Stanford, CA 94305-2125

finn@math.stanford.edu

Kefeng Liu

Department of Mathematics

University of California

Los Angeles, CA 90095-1555

liu@math.ucla.edu
Darren Long

Department of Mathematics

University of California

Santa Barbara, CA 93106-3080

long@math.ucsb.edu

Jiang-Hua Lu

Department of Mathematics

The University of Hong Kong

Pokfulam Rd., Hong Kong jhlu@maths.hku.hk

Alexander Merkurjev

Department of Mathematics University of California

Los Angeles, CA 90095-1555 merkurev@math.ucla.edu
Sorin Popa

Department of Mathematics

University of California

Los Angeles, CA 90095-1555

popa@math.ucla.edu

Jie Qing

Department of Mathematics

University of California

Santa Cruz, CA 95064

qing@ cats.ucsc.edu

Jonathan Rogawski

Department of Mathematics

University of California

Los Angeles, CA 90095-1555

jonr@math.ucla.edu

\section{PRODUCTION}

pacific@math.berkeley.edu

Silvio Levy, Scientific Editor Matthew Cargo, Senior Production Editor

ACADEMIA SINICA, TAIPEI

CALIFORNIA INST. OF TECHNOLOGY

INST. DE MATEMÁTICA PURA E APLICADA

KEIO UNIVERSITY

MATH. SCIENCES RESEARCH INSTITUTE

NEW MEXICO STATE UNIV.

OREGON STATE UNIV.

\section{SUPPORTING INSTITUTIONS}

STANFORD UNIVERSITY
UNIV. OF BRITISH COLUMBIA
UNIV. OF CALIFORNIA, BERKELEY
UNIV. OF CALIFORNIA, DAVIS
UNIV. OF CALIFORNIA, LOS ANGELES
UNIV. OF CALIFORNIA, RIVERSIDE
UNIV. OF CALIFORNIA, SAN DIEGO
UNIV. OF CALIF., SANTA BARBARA

UNIV. OF CALIF., SANTA CRUZ

UNIV. OF MONTANA

UNIV. OF OREGON

UNIV. OF SOUTHERN CALIFORNIA

UNIV. OF UTAH

UNIV. OF WASHINGTON

WASHINGTON STATE UNIVERSITY

These supporting institutions contribute to the cost of publication of this Journal, but they are not owners or publishers and have no responsibility for its contents or policies.

See inside back cover or www.pjmath.org for submission instructions.

The subscription price for 2011 is US \$420/year for the electronic version, and \$485/year for print and electronic.

Subscriptions, requests for back issues from the last three years and changes of subscribers address should be sent to Pacific Journal of Mathematics, P.O. Box 4163, Berkeley, CA 94704-0163, U.S.A. Prior back issues are obtainable from Periodicals Service Company, 11 Main Street, Germantown, NY 12526-5635. The Pacific Journal of Mathematics is indexed by Mathematical Reviews, Zentralblatt MATH, PASCAL CNRS Index, Referativnyi Zhurnal, Current Mathematical Publications and the Science Citation Index.

The Pacific Journal of Mathematics (ISSN 0030-8730) at the University of California, c/o Department of Mathematics, 969 Evans Hall, Berkeley, CA 94720-3840, is published monthly except July and August. Periodical rate postage paid at Berkeley, CA 94704, and additional mailing offices. POSTMASTER: send address changes to Pacific Journal of Mathematics, P.O. Box 4163, Berkeley, CA 94704-0163.

PJM peer review and production are managed by EditFLOW ${ }^{\mathrm{TM}}$ from Mathematical Sciences Publishers.

PUBLISHED BY PACIFIC JOURNAL OF MATHEMATICS

at the University of California, Berkeley 94720-3840

A NON-PROFIT CORPORATION

Typeset in IATEX

Copyright $(2011$ by Pacific Journal of Mathematics 


\section{PACIFIC JOURNAL OF MATHEMATICS}

Volume $249 \quad$ No. $2 \quad$ February 2011

A gluing construction for prescribed mean curvature

257

ADRIAN BUTSCHER

Large eigenvalues and concentration

271

BRUNO COLBOIS and ALESSANDRO SAVO

Sur les conditions d'existence des faisceaux semi-stables sur les courbes multiples primitives

JEAN-MARC DRÉZET

A quantitative estimate for quasiintegral points in orbits

LIANG-CHUNG HSIA and JosePh H. Silverman

Möbius isoparametric hypersurfaces with three distinct principal curvatures, II

ZEJUN HU and SHUJIE ZHAI

Discrete Morse theory and Hopf bundles

371

DMITRY N. KOZLOV

Regularity of canonical and deficiency modules for monomial ideals

ManOJ KUMmini and SATOSHI MURAI

$\mathrm{SL}_{2}(\mathbb{C})$-character variety of a hyperbolic link and regulator

WEIPING LI and QINGXUE WANG

Hypergeometric evaluation identities and supercongruences

LING LONG

Necessary and sufficient conditions for unit graphs to be Hamiltonian

H. R. Maimani, M. R. Pournaki and S. Yassemi

Instability of the geodesic flow for the energy functional

DOMENico PERrone

String structures and canonical 3-forms

CORBETT REDDEN

Dual pairs and contragredients of irreducible representations

BINYONG SUN

On the number of pairs of positive integers $x_{1}, x_{2} \leq H$ such that $x_{1} x_{2}$ is a $k$-th power

DOYCHIN I. TOLEV

Correction to the article A Floer homology for exact contact embeddings 